\title{
AUTO-PERCEPCIÓN DEL PROFESORADO SOBRE SU FORMACIÓN EN EDUCACIÓN EMOCIONAL
}

Iratxe Suberviola-Ovejas': Universidad de la Rioja. España

iratxe.suberviola@unirioja.es

\section{RESUMEN}

Las últimas investigaciones en el ámbito emocional apuntan como, muchas de las características socio-educativas que presenta el alumnado de los PCAs están íntimamente relacionadas con la eficacia en competencias emocionales. Esto pone de manifiesto la conveniencia de introducir programas de educación emocional, con docentes formados al respecto. El objetivo de este estudio es mostrar la brecha existente, en cuanto a la formación y la designación del profesorado de los itinerarios no-ordinarios, y la idoneidad percibida, por parte de este colectivo, sobre dichos aspectos.

PALABRAS CLAVE: Educación emocional - Inteligencia emocional - Itinerarios noordinarios - Formación del profesorado

\section{PERCEPTION OF THE TEACHERS ABOUT TRAINING IN EMOTIONAL EDUCATION}

\begin{abstract}
Recent researchs on emotions say that many of the socio-educational characteristics of non-ordinary itinerary students are related to emotional competencies effectiveness. It shows the convenience of introducing emotional education programs with teacher. The aim of this study is to show the difference between; the formation and the designation of the teachers of the PCAs, and the perceived suitability, by this collective, about these aspects.
\end{abstract}

KEY WORDS: Emotional education - Emotional intelligence - Non-ordinary itinerary - Teacher training

\footnotetext{
${ }^{1}$ Autor correspondiente

Iratxe Suberviola Ovejas: ontratada interina, Departamento Ciencias del Educación. Universidad de la Rioja, España.

Correo: iratxe.suberviola@unirioja.es
} 


\section{INTRODUCCIÓN}

La Organización Mundial de la Salud define a los seres humanos como seres biopsico-sociales, lo que apunta la conjunción en una persona de mente y cuerpo y la relación entre estas. A su vez, tanto lo biológico como lo psicológico, influyen en nuestras relaciones y comportamientos en sociedad. En este sentido, existen numerosos estudios que relacionan la inteligencia emocional y la eficacia en diferentes competencias emocionales con el bienestar y el equilibrio personal. (Extremera \& Duran, 2006, entre otros) Por otro lado, también existe vinculación entre las emociones y el aspecto biológico. La experimentación de ciertas emociones produce modificaciones a nivel fisiológico como; aumento de la actividad en el hipotálamo, aumento de reactividad gastrointestinal, etc. (Fernández-Abascal \& Chóliz, 2006).

Pero, en la unión entre lo emocional y lo biológico, no debemos referirnos únicamente a la más que afirmada relación existente entre cuerpo y mente, y abogada por psicólogos, educadores, nutricionistas, psiquiatras, médicos y otras profesiones, sino a la relación comprobada en conductas como el consumo de sustancias adictivas y/o ilegales y las competencias emocionales de los adolescentes. (Ruiz- Aranda et. al, 2010; Trinidad \& Johnson, 2002, entre otros).

Cuerpo y mente marcan y condicionan las relaciones y comportamientos sociales de las personas, de lo que se deriva la existencia de una relación directa entre las emociones y ciertas variables socio-educativas. Concretamente en los alumnos se ha detectado conexión entre la eficacia emocional y las relaciones interpersonales. (Extremera \& Fernández-Berrocal, 2004a, 2004b; Lopes, Salovey \& Strauss, 2003, entre otros) La literatura también determina la relación de la emotividad con el rendimiento académico y la actitud hacia el aprendizaje (Fernández-Berrocal \& Extremera, 2006; Fernández- Berrocal, Extremera \& Ramos, 2004, entre otros) y de ésta con las conductas disruptivas en el aula. (Extremera \& Fernández-Berrocal, 2004c, entre otros).

\subsection{Características del alumnado de los itinerarios no-ordinarios}

Aunque en los primeros momentos la unión entre lo cognitivo y el rendimiento académico era lineal, actualmente está demostrado que esta relación no siempre se cumple y que, en ocasiones, alumnos con capacidades cognitivas adecuadas presentan un bajo rendimiento académico, influenciado, en cierta medida, por la actitud que este alumnado pueda presentar hacía el aprendizaje y el sistema académico. Mucho de este alumnado está escolarizado en itinerarios no-ordinarios como son; los programas de cualificación profesional inicial (PCPI) o los programas de currículo adaptado (PCA).

El alumnado de estos programas habitualmente presenta las siguientes características: valora negativamente el marco escolar; presentan serias dificultades de adaptación; 
buscan en las actividades resultados inmediatos y aplicables, presentan baja tolerancia a la frustración; acumulan un retraso escolar que hace muy difícil su desarrollo educativo en un grupo ordinario; manifiestan dificultades de convivencia y serios problemas de conducta en el ámbito escolar. Además de estas características, la realidad apunta que en este alumnado se encuentran variables como el consumo de sustancias adictivas, el desequilibrio personal, las conductas disruptivas, los comportamientos agresivos y prácticas de riesgo, en mayor medida que en el alumnado de las aulas ordinarias.

\subsection{Educación emocional en los itinerarios no-ordinarios}

Estas particularidades fundamentan que en estos programas, además de los objetivos puramente académicos, se deban desarrollar otros como: obtener niveles adecuados de socialización y convivencia; propiciar la madurez humana propia de etapa de secundario y garantizar las competencias básicas para el desenvolvimiento en la vida activa. Estos objetivos contemplan intrínsecamente la conveniencia de incluir la educación emocional en el currículo, puesto que la finalidad educativa de estos programas tiene que tener un carácter compensador a la vez que preventivo.

En relación al carácter profiláctico que debe marcar la educación de estos alumnos, en la literatura se proponen dos categorías de factores preventivos sobre los agentes de riesgo que inciden en un desarrollo bio-psico-social inadecuado, contraproducente, y peligroso de los individuos. Estos factores pueden agruparse en personales y ambientales. (Graczyk et al., 2000) Las competencias socio-emocionales se sitúan como características personales que ejercen de factores preventivos. Dentro de estas se encuentran: las habilidades sociales, las habilidades de solución de problemas interpersonales, la autoeficacia, la autoestima, las habilidades de comunicación efectiva y las aspiraciones elevadas. Los factores ambientales que aportan un contexto de apoyo y favorecen el desarrollo social y emocional del joven son: el compromiso fuerte con al menos un adulto, el comportamiento apropiado de los padres, la implicación en organizaciones constructivas, las actividades sociales y el acceso a una educación óptima.

Los docentes de secundaría, en contadas ocasiones pueden intervenir para modificar los factores ambientales. Sin embargo, tienen la posibilidad y la obligación de que los centros educativos aporten a los alumnos estrategias que les ayuden a desarrollar habilidades sociales, que les orienten sobre la resolución de conflictos, que les guíen en el desarrollo de un identidad realista y ajustada, que favorezca la autoestima, etc.

Si todos estos factores son importantes en los alumnos de las aulas ordinarias, en el alumnado de itinerarios no-ordinarios se tornan imprescindibles. El desarrollo de los factores socio-emocionales como factores preventivos, junto con la relación existente entre las diferentes variables socio-educativas y la eficacia en las competencias educativas, evidencian la importancia de la inclusión de la educación emocional de un modo global en el currículo y, no únicamente de un modo anecdótico o puntual. La educación emocional se presenta como una respuesta útil $\mathrm{v}$ necesaria para 
abordar esta problemática, dentro de la concepción de educación compensatoria y preventiva que debe estar presentes en los PCAs y PCPIs, como una forma de prevención primaria inespecífica, entendiendo como tal, la adquisición de competencias que se pueden aplicar a la multiplicidad de situaciones tales como la prevención del consumo de drogas, prevención del estrés, ansiedad, depresión, violencia, etc. (Bisquerra, 2005).

Este estudio parte de la concepción de Educación Emocional propuesta por Bisquerra (2005) afirmando que es "un proceso educativo, continuo y permanente, que pretende potenciar el desarrollo de los competencias emocionales como elemento esencial del desarrollo integral de la persona, con objeto de capacitarle para la vida. Todo ello tiene como finalidad aumentar el bienestar personal y social". (Bisquerra, 2005 p.2).

En este sentido partimos de que la puesta en práctica de un programa de educación emocional requiere una formación previa del profesorado. Sin embargo, el desarrollo de competencias emocionales de un modo intencional y sistemática está, en general, bastante ausente en los programas de formación de maestros, (Bisquerra, 2005) tanto desde un punto de vista de la formación inicial, como en la formación continua del mismo. La importancia de esta formación docente se acrecienta en el profesorado de los itinerarios no-ordinarios, debido a las características propias del alumnado.

No debemos olvidar que el proceso de enseñanza-aprendizaje se produce a través de una interacción educativa entre el docente y el alumnado que no es emocionalmente neutra. En este sentido existe un estudio desarrollado por Bernal y Cárdenas (2009) que desvela que las interacciones entre docentes y discentes se encuentran impregnadas por la afectividad, reflejándose en las huellas mnemónicas de los episodios escolares donde se percibe un alto componente afectivo o emocional. La afectividad desarrollada por lo profesores podría ser un posible anclaje para la vinculación afectiva de los alumnos hacia el aprendizaje, la motivación y el desarrollo individual. Del mismo modo, las experiencias emocionalmente negativas vividas en la relación profesor-alumno causaría una desmotivación y distanciamiento hacía el aprendizaje.

Para que el alumno desarrolle unas adecuadas competencias emocionales necesita de un educador emocional. El docente, lo quiera o no, es un agente activo de desarrollo afectivo y debería hacer un uso consciente de estas habilidades en su trabajo puesto que es un modelo adulto a seguir por sus alumnos en tanto son la figura que posee el conocimiento, pero también la forma ideal de ver, razonar y reaccionar ante la vida, convirtiéndose en un modelo de inteligencia emocional insustituible, por lo que, además de la enseñanza de conocimientos teóricos, le corresponde moldear y ajustar en el aula el perfil afectivo y emocional de sus alumnos. (Extremera \& FernándezBerrocal, 2004c).

El profesor José M. Esteve, (2006) después de trabajar durante más de dos décadas con circuitos cerrados de televisión en el contexto escolar afirma aue el clima emocional 
del aula lo construye el profesor a partir de dos elementos básicos: 1) La identidad profesional con la que afronta la relación educativa; es decir, la definición personal que hace de su propio papel como profesor desde el cual enfoca las relaciones con sus alumnos con distintas actitudes y estrategias; 2) Las destrezas de interacción y comunicación con los que el docente afronta la relación educativa. Este autor afirma que las posibles actitudes negativas de los alumnos son reactivas, es decir, se elaboran con los sentimientos y actitudes que los profesores producen desde el primer día de clase, generando respeto o rechazo, a partir de la lectura emocional que los alumnos hacen de las actitudes y sentimientos mostrados por los docentes.

Del mismo modo, desde el mismo momento en que se producen las reacciones emocionales por parte de los alumnos, éstas se convierten en un elemento más de la relación educativa, ya que los docentes las captan inmediatamente, a partir de la comunicación no verbal y gestual de los alumnos, a partir de donde se inician una cadena imparable de acción-reacción que, en muchos casos está en la génesis de las reacciones agresivas de los discentes y de las actuaciones de los profesores.

Que el profesorado conozca cómo influyen sus actitudes, emociones y comportamientos hacía el alumnado va a proporcionar una base teórica sobre cómo actuar con los estudiantes y va a dotarles de mecanismos de intervención para la mejora de las relaciones en el aula, para fomentar la motivación hacía el aprendizaje. Esta necesidad se acentúa en los itinerarios no ordinarios debido a las problemáticas sociales tanto entre iguales como entre profesor-alumno que de forma habitual se producen.

\section{METODOLOGÍA}

\subsection{Objetivos}

El objetivo principal de este estudio es averiguar el juicio del profesorado, de los itinerarios no-ordinarios, sobre la idoneidad formativa y organizativa de los docentes de los PCAs y PCPIs. Para ello se estipulan los siguientes objetivos específicos: 1) Mostrar cuál es la percepción del profesorado de los itinerarios no-ordinarios sobre la formación idónea para desarrollar la labor educativa en estas aulas; 2) Analizar la percepción que los docentes de los itinerarios no-ordinarios presentan sobre su formación para desarrollar las competencias emocionales de sus alumnos.

\subsection{Participantes}

Esta pesquisa se lleva a cabo mediante un diseño de muestra intencional, puesto que dentro del objetivo está el llegar a un conocimiento o teoría a través del análisis de los datos obtenidos. Dentro de éste, la selección de la muestra se produce mediante la combinación de ambos métodos; el muestreo opinativo y el muestreo teórico, puesto que por un lado, se propone como sujetos a ser entrevistados aquellos que consideramos más idóneos y representativos como conocedores de los itinerarios noordinarios v por otro lado, se considera que es conveniente no cerrar el número de 
sujetos a entrevistar sino que es más rico poder modificar los mismos a partir de la obtención y análisis de datos.

Los docentes entrevistados pertenecen a 4 centros de Navarra de diferentes comarcas: (Se omite el nombre con objeto de mantener el anonimato) 1) Centro de la comarca de Tierra Estella. Se trata de un centro de secundaria y ballicher de carácter concertado. Cuenta con una única aula de PCA. Dentro de este centro son entrevistados: La tutora de la PCA; El director académico; Un/una docente del área científico-matemático; Un profesor/a del área de Educación Física; Un/una docente del área socio-lingüística. (Esta entrevista fue eliminada en el proceso de validación por carecer de los parámetros de calidad marcados); Un/una docente del ámbito práctico; 2) Centro de la comarca de la Ribera Baja. Se trata de dos aulas externas de PCA, cuyo alumnado es dependiente de un centro público de educación secundaria a nivel burocrático. La gestión de dichas aulas es particular de este centro y el carácter es concertado. Todos los docentes del centro son tutores puesto que dichas figuras no están establecidas por aula sino por alumnos, de modo que cada profesor es tutor de un número de discentes.

La totalidad de los docentes de este centro han sido entrevistados: Dos docentes del ámbito científico-matemático; Un miembro del equipo directivo; Dos docentes del ámbito socio-lingüístico; Un/una profesor/a del ámbito práctico; 3) Centro de la comarca de la Merindad de Pamplona. Se trata de un centro concertado que cuenta con dos aulas de PCA y varias aulas de PCPI de cocina. Un importante número de alumnos de los programas de currículo adaptado tienen carácter de internos. La totalidad de los docentes de este centro han sido entrevistados: Los dos tutores de ambas aulas de PCA; El director del centro educativo; Un docente del ámbito científico-técnico; Un docente del ámbito socio-lingüístico; La orientadora del centro; Un docente del ámbito práctico; 4) Centro de la comarca de la Ribera Alta. Se trata de un centro público de educación secundaria y ballicher que cuenta con un aula de PCA y varios PCPIs. Los agentes educativos que forman parte de la muestra son: La orientadora y responsable de convivencia; Un docente del ámbito científicomatemático; Un docente del ámbito socio-lingüístico; Dos docentes del ámbito práctico; El tutor del PCA.

\subsection{Procedimiento}

La recogida de datos de este estudio se lleva a cabo a través de entrevistas de tipo individual, holística y no estructurada. Las entrevistas se realizan teniendo en consideración las directrices aportadas por Ruiz-Olabuénaga (2007) sobre el proceso de interacción y sonsacamiento. El proceso de recogida de datos se realiza a través de la grabación en audio digital y la posterior transcripción literal de la totalidad de la entrevista.

Los códigos establecidos en un primer momento, para las personas entrevistadas responden a: 
a)

Zonificación de Navarra: Merindad de Pamplona (Mp), Ribera Baja (Rb), Ribera Alta (Ra), Tierra Estella (Te).

b)

Tipología de aulas: aulas internas insertadas en centros de educación secundaria (i); aulas externas, independientes a centros de educación secundaria (e).

c) Número de años de experiencias en itinerarios no-ordinarios: Se escribe el número de años.

d) Ámbito de labor educativa: ámbito socio-lingüístico (sl), ámbito científico- matemático ( $\mathrm{cm})$, ámbito práctico $(\mathrm{p})$, tutor $(\mathrm{t})$, gestión $(\mathrm{g})$.

Posteriormente, tras el análisis de las primeras entrevistas, se comprobó que el discurso no variaba ni entre el profesorado de las diferentes zonas, ni con respecto al ámbito educativo en el que estuvieran desarrollando la docencia. Sin embargo, sí se pudo apreciar diferencias entre el contenido, en los que refiere a la figura contractual de los profesores.

La codificación de esta segunda fase sería la siguiente:

a)

Número de entrevista: Se pone en número cardinal la posición en la que se realizó la entrevista

b)

Tipología de aulas: aulas internas insertadas en centros de educación secundaria (i); aulas externas, independientes a centros de educación secundaria (e).

c) Número de años de experiencias en itinerarios no-ordinarios: Se escribe el número de años.

d) profesorado interina (i), personal definitivo (d).

Figura contractual:

El proceso de categorización se desarrolla a través de un análisis mixto (deductivoinductivo) en el que existe una primera parte en la que dichas categorías surgen a partir del análisis de la literatura existente al respecto y, un segundo momento en el que estas categorías son cumplimentadas por el análisis de la información derivada de las entrevistas.

A continuación se muestran las categorías y sub-categorías pertenecientes al ámbito de la formación docente en los itinerios no-ordinarios. Dentro de éste se incluye tanto la realidad formativa del profesorado de las PCAs como la idoneidad que, los mismos entrevistados, perciben que debe tener el personal encargado de educar a este tipo de alumnado:

a)

Realidad formativa - Alude a la formación que presentan los sujetos; tanto la formación inicial como los complementos formativos realizados. Esta sub-categoría se divide en dos sub- 
categorías: o Formación inicial - Se refiere a la titulación que permite a los entrevistados ejercer su labor educativa dentro de los PCA.

b)

Complementos formativos - Hace referencia a aquellos cursos, materias, disciplinas, máster, postgrados, experiencia, etc., que hayan realizado los entrevistados.

c)

Idoneidad formativa - Se trata de la percepción de los entrevistados sobre la formación idónea del profesorado de los itinerarios no-ordinarios, tanto en lo que respecta a la formación inicial como a aquellos complementos formativos de interés para ejercer la labor educativa.

d)

refiere a la percepción que tienen los entrevistados sobre qué tipo de formación inicial debe tener el profesorado de estos programas, es decir, cuales son los titulaciones idóneas de acceso para el profesorado de los itinerarios noordinarios. También se contempla dentro de esta sub-categoría las modificaciones que se consideran aconsejables introducir en dichas titulaciones.

e)

formativos- Se trata de los complementos a las titulaciones de acceso a la docencia de los PCAs y PCPIs que se consideran idóneas para la realización de la labor educativa dentro de estos grupos. Formarían parte de esta subcategoría, cursos, máster, posgrados, especialidades, formación entre el profesorado, formación laboral, etc.

\section{ANÁLISIS Y DISCUSIÓN}

\subsection{Realidad formativa. Formación inicial}

Se aprecia que existe gran disparidad en las titulaciones. No obstante, es importante destacar que se observan diferencias entre los docentes de los programas externos e internos en lo que se refiere a titulaciones. El profesorado perteneciente a las aulas externas presenta una tipología de preparación inicial más relacionada con el ámbito socio-educativo, con titulaciones como; la pedagogía, psicología, educadores sociales, sociólogos, etc. Los docentes de los programas internos presentan titulaciones diversas, que en su mayoría poco tienen que ver con el ámbito socio-pedagógico. Un total de 9 de los 13 docentes entrevistados de los itinerarios no-ordinarios externos tienen titulaciones con este perfil, mientras que únicamente 2 de los entrevistados de las aulas internas lo presentan.

\subsection{Realidad formativa. Complementos formativos}

Dentro de esta sub-categoría encontramos diversos ámbitos en cuanto a los complementos formativos desarrollados por los docentes. El primero de éstos y, a la vez el más valorado, es la experiencia de cada individuo tanto en la docencia en los propios programas como en otra tipología de actividades socio-educativas similares. Prácticamente, la totalidad de los entrevistados enfatizan la importancia de la 
experiencia para poder ejercer la labor educativa efectiva y afectiva con este tipo de alumnado.

¿Qué me ha formado? La gente, a mí valoro más cuestiones de experiencia de la gente, de estar al lado de la gente, más que a lo mejor cursos que han podido servir más como herramientas pero cursos teóricos, de profundizar en esta cuestión sobre el fracaso escolar o chavales con ciertas características de las que ya se llaman chavales en conflicto social, pues muy poquitos. (e.8.d)

Otra de las temáticas encontradas dentro de esta sub-categoría hace referencia a la escasa y poco definida oferta formativa relacionada, tanto con la educación emocional en general, como dirigida al alumnado de los itinerarios no-ordinarios en concreto.

Acabo de hacer un curso en Pamplona con un título muy atractivo en relación a atención, herramientas en el aula para atención a la diversificación y no sé; como estábamos pues claro, una profesora que es muy conductista y todas sus soluciones son muy conductistas, cosa que puede estar bien, pero que no puede ser para todo el mundo. (12.e.8.d)

El complemento formativo menos valorado por la totalidad de los docentes que lo han realizado, es el Curso de Adaptación Pedagógica (CAP).

Ni para esto ni para ser profesor (...) te enseñan lo que es la Ley. Las prácticas son de risa porque yo fui a un instituto de Logroño y estuve sentado en la silla durante quince días y el profesor dio la clase (...) creo que no te preparan para esto. (21.i.1.i)

Uno de los recursos formativos más utilizados por los docentes de los itinerarios noordinarios, especialmente por aquellos de las aulas externas que carecen de una formación inicial socio-educativa relacionada con alumnado con este perfil, es la consulta de bibliografía, estudios o proyectos educativos.

Cuando empezó la idea de empezar a hacer, a trabajar en un proyecto para adolescentes, que no sabíamos si iba a ser aquí o en donde, empezamos a formarnos también un poco en la temática en concretos y a los adolescentes en riesgo y tal ¿no?... (¿y dónde os formasteis?)...Pues con bibliografía de gente que estaba estudiando...(¿autodidactas?)...Sí, sí, total, total, sí. (17.e.10.d) 
Otro del ámbito de formación del profesorado es a través del intercambio de conocimientos, experiencias, recursos, opiniones, etc., entre los agentes educativos de estos itinerarios.

"Los último dos años sí que se está haciendo unas reuniones con los PCAs, donde se invita a los PCAs y sobre todo más que cursos de; "viene una persona y te doy un formación" es un poco entre nosotros [...] se hace una serie de preguntas y entonces un poco son experiencias, compartimos experiencias que a lo mejor a mí me han servido (...) los expongo por si a otra PCA les puede ayudar. (6.e.8.d)

\subsection{Idoneidad formativa. Formación inicial}

Una de las corrientes detectadas en los docentes es la conveniencia de que se introduzcan contenidos específicos relacionados con este tipo de aulas y de perfil de alumnado en la formación inicial del profesorado.

Unas asignaturas, no sé cómo se podría poner, pero sí que habría que tener una formación diferencial a la Primaria (...) porque es que sino al final los educadores, los profesores y las profesoras no me extraña que se quemen y que se estresen y se cojan bajas, (...) tiene que ser duro. (6.e.8.d)

Otra de las vertientes que apunta el profesorado entrevistado es la especificidad de la formación docente en referencia a los itinerarios alternativos. Consideran que la introducción de unas asignaturas y/o contenidos relacionados con este perfil de alumnos puede ser escaso, por los se propone una especialización dentro de la formación inicial del profesorado enfocada a este tipo de itinerarios.

(¿Consideras importante que hubiera una especialidad o...?) Yo creo que sí, sí porque además cada vez nos estamos. El sistema educativo cada vez está haciendo la creación de la diversificación, los PCPIs, el tema de la PCA, o sea, que va aumentando el número de este tipo de grupos específicos. (16.i.8.d)

Una de las sugerencias más innovadoras es la realizada por un profesor con respecto a la formación inicial del profesorado, en concreto en el periodo de prácticas. La propuesta se basa en la supervisión de dichos alumnos por personal experto en el que se realiza un proceso formativo, de autoreflexión y autoconocimiento sobre lo que el alumno de prácticas siente, piensa, cómo reacciona y por qué lo hace.

... para plantear el tema de la supervisión como espacios de reflexión para los chavales que están en prácticas [...] espacios de reflexión que son los procesos de supervisión que nosotros hacemos (...) espacios donde pueden reflexionar y donde se le pueda acompañar a darse cuenta, hacerse consientes de qué es 
lo que les está pasando (...), ¿qué le pasa a él?, ¿cuál es la respuesta que da?, ¿por qué la da?, ¿cómo reaccionamos cuando nos sentimos atacados?. (3.e.11.d)

Un grupo de docentes realiza una crítica más amplía sobre el sistema educativo en el que se afirma que, no únicamente no está bien orientada la formación inicial para estos grupos específicos, sino que existen otras deficiencias más genéricas que se relacionan, en muchos casos, con el concepto de educación. Se realiza un reproche con respecto a la brecha existente entre el discurso y el concepto sobre educación presente en algunos foros del ámbito académico, en el que se hace hincapié en la inclusión de la educación emocional, social, en valores, etc., como finalidades fundamentales de la educación de los alumnos, y las posteriores prácticas educativas. Esta fisura se refiere tanto a la formación inicial del profesorado como a los currículos que se están desarrollando en las aulas.

No sólo en lo emocional, emocional, socioemocional, toda una...Sí, yo creo que estamos en un país que estamos siempre repitiéndonos a nosotros mismos que la formación inicial del profesorado tendría que ser más rica (...) Sí, es cierto que hay un discurso (...) pero yo creo que no está encarnado en lo que es la formación, no es una cosa que está cristalizada ni en la formación inicial, ni en el currículum, ni...(22.e.13.d)

\subsection{Idoneidad formativa. Complementos formativos}

Uno de los ámbitos de formación mejor valorados por las personas entrevistadas es la iniciativa creada por la administración para realizar unos encuentros periódicos entre los agentes formativos de los PCAs. "... en Navarra hay una iniciativa que por lo menos es interesante, (...) que es un equipo desde el departamento principalmente en los PCAs, y sí que están dando algo de movimiento a este curso. (15.e.2.d)

Otro de los aspectos en los que el profesorado pone el acento es en el funcionamiento de los equipos de los itinerarios alternativos. La unión y apoyo entre los agentes educativos como modo de formación y aprendizaje de la experiencia y conocimientos de otros compañeros.

Entonces yo lo que intentaría sería que fuesen profesionales preparados y luego que hubiese un equipo, un equipo porque adonde tú no llegues el equipo te va a llevar. Y si hace falta formar en los centros, en las PCAs internas, equipos para PCAs o PCPIs. (14.e.11.d)

La formación complementaria en educación emocional es considerada por los profesores entrevistados como valiosa y sustancial en los docentes que desarrollan su labor educativa con alumnado con este perfil concreto. "Para ellos es necesario un profesor emocional [...] En los CAPs no hay (...) y tampoco creo que sea algo muy demandado, creo que no se le da todo el valor a la educación emocional como se debería dar". (1.i.11d) 
Partiendo de la evidencia y empíricamente constatada influencia que la eficacia en competencias emocionales presenta en aspectos como; los educativos, el desarrollo personal, las relaciones sociales y la salud física y psíquica, la educación socioemocional se hace prioritaria en alumnado con perfil propio de los itinerarios no ordinarios. Para llevar a cabo este cometido es preciso poseer formación previa.

En este estudio se ha evidenciado como los docentes, especialmente en los programas internos, carece de formación inicial socio-pedagógica, con titulaciones diversas poco relacionadas con el ámbito educativo. En muchos casos la única formación en el ámbito educativo ha sido el Curso de Adaptación Pedagógica que, la totalidad de los entrevistados lo califica como poco útil para la posterior docencia, especialmente con esta tipología de alumnado. La inexistencia de una formación socio-educativa previa va a dificultar sobremanera el desarrollo de una labor educativa en la que se incluyan de un modo eficaz los objetivos y contenidos en educación emocional.

Los docentes que poseen una formación inicial relacionada con el ámbito sociopedagógico, afirman que dichos estudios presentaban carencias en aprendizajes sobre educación emocional y en aspectos como la resolución de conflictos o la educación para la convivencia. No obstante, a menudo estos docentes presentan complementos formativos en esta línea y valoran la inclusión de la educación emocional en los itinerarios no-ordinarios como imprescindible. Además, presentan mayor control de la situación manteniéndose más serenos y equilibrados ante situaciones "amenazantes", tal como expone. (Esteve, 2006)

\section{CONCLUSIONES}

Vista la necesidad y conveniencia de la educación emocional, tanto de cara a la transmisión a los estudiantes como de cara a la competencia para la docencia en este tipo de programas, ésta debería incluirse en la formación inicial del profesorado.

Los docentes de los itinerarios no-ordinarios realizan propuestas para la formación docente en temáticas relacionados con; la educación emocional, la educación para la convivencia y la formación específica para este tipo de alumnado. Sus planteamientos están orientados a la formación inicial del profesorado como a la formación continua.

En la formación inicial las sugerencias son las siguientes:

a)

La inclusión de la educación emocional como uno de los módulos básicos en los Grados de magisterios de Infantil y Primaria. Esto se fundamenta en el carácter preventivo que presenta dicha educación. Esto hace que se deba intervenir desde edad temprana para el correcto desarrollo de las competencias socio-emocionales, por ello es sustancial la inclusión de la educación emocional en la formación del profesorado de estos Grados. Se debe tener presente que la titulación mínima exigida para la docencia en los PCAs es de Grado de magisterio en Primaria. 
b)

Dentro del módulo de optatividad se debería ofertar una serie de asignaturas que otorgasen la "Mención en educación de itinerarios no- ordinarios". Éstas estarían relacionadas con jóvenes en situación de riesgo, educación para la convivencia y educación emocional. Esta medida formaría docentes especializados en este tipo de itinerarios y de alumnado.

c)

En el módulo de prácticum se propone la inclusión de la supervisión experta dentro de las prácticas de magisterio, donde se forme al alumno en su gestión emocional. Para ello se le debe instruir cómo desarrollar la labor docente desde la autoreflexión y el autocontrol emocional consciente, dirigido a la creación de un clima adecuado en el aula y al desarrollo de las competencias emocionales en los discentes.

d)

Dentro del Master universitario de educación secundaria se sugiere una especialización para los itinerarios no-ordinarios donde, se introduzcan asignaturas referentes a las características particulares de los adolescentes en riesgo de exclusión, educación para la convivencia, educación emocional, herramientas para fomentar la motivación y la autoestima, etc.

Las proposiciones para la formación continúan del profesorado de los itinerarios noordinarios son las siguientes:

a)

Se perciben como muy positivos los encuentros entre agentes educativos de itinerarios no-ordinarios con objeto de compartir experiencias, herramientas, recursos $u$ opiniones. Se sugiere aprovechar estos foros para la realización de cursos impartidos por personal experto en educación emocional, resolución de conflictos, educaciones para la convivencia y otras temáticas relacionadas con estos programas alternativos.

b)

Se considera aconsejable la creación de equipos docentes de profesorado de itinerarios no-ordinarios donde se debatan las finalidades, objetivos y contenidos educativos con estos grupos y las herramientas para llevarlas a cabo. Estos equipos servirán de apoyo y orientación para los docentes que lo demanden.

\section{REFERENCIAS}

Bernal, A \& Cárdenas, A.R. (2009). Influencia de la competencia emocional docente en la formación de procesos motivacionales e identitarios en estudiantes de educación secundaria. Revista de Investigación Educativa, 2009, 27 (1). Pag. 203-222.

Bisquerra, R. (2005). La educación emocional en la formación del profesorado. Revista Interuniversitaria de Formación del Profesorado, 19 (3):95-114.

Esteve, J.M. (2006). Las emociones en el ejercicio práctico de la docencia. Teoría Educativa. 18: 85-107. 
Extremera, N \& Duran, A. (2006). Inteligencia emocional y su relación con los niveles de burnout, engagement y estrés en estudiantes universitarios. Revista de Educación, (342):239-256

Extremera, N \& Fernández-Berrocal, P. (2004a). El papel de la inteligencia emocional en el alumnado: evidencias empíricas. Revista eléctrónica de inteligencia educativa. 6 (2)

Extrenera, N \& Fernández-Berrocal, P. (2004b). Inteligencia emocional, calidad de las relaciones interpersonales y empatía en estudiantes universitarios. Clínica y salud, 15(2):117-137.

Extremera, N \& Fernández-Berrocal, P. (2004c). La inteligencia de desarrollar la inteligencia emocional en el profesorado. Revista Iberoamericana de Educación, 33(8).

Fernández Abascal, E \& Chóliz, M. (2006). La cara espejo de las emociones. Madrid: UNED. Universidad Nacional de Educación a distancia.

Fernández Berrocal, P \& Extremera, N. (2006): “Emotional Intelligence: A theoretical and empirical review of its first 15 years of history. Psicothema, (18): 7-18.

Fernández- Berrocal, P., Extremera, N \& Ramos, N. (2004).Validity and reliability of the Spanish Modified Version of the Trait Mete-Mood Scale. Psychological Reports, (94):751-755.

Graczyk, P.A, Weissberg, R.P., Payton, J.W., Elias, M.J., Greenberg, M.T \& et al. (2000). The Handbook of Emotional Intelligence. Theory Development, Assessment, and Aplication at Home, School, and in the Workplace.S. Francisco: Jossey-Bass.

Lopes, P. N., Salovey, P \& Straus, R. (2003). Emotional intelligence, personality and the perceptive quality of social relationships. Personality and Individual Differences, 35(3):641-658.

Ruiz Aranda, D., Cabello, R., Martín Salguero, J., Castillo, R., Extremera, N \& et al. (2010). Los adolescentes Malagueños ante las drogas: la influencia de la Inteligencia Emocional. Málaga: UNESCO.

Ruiz Olabuenaga, J.I. (2007). Metodología de la investigación cualitativa. Bilbao: Universidad de Deusto

Trinidad, D.R \& Jhonson, P. (2002). The association between emotional intelligence and early adolescenc et tobacoo and alcohol use. Personality and Individual Differences, 32(1):95-105.

\section{Iratxe Suberviola-Ovejas}

Lda en Psicopedagogía, con el Diploma de Estudios Avanzados en la modalidad de Didáctica y Organización Escolar. Profesora del área de Teoría e Historia de la 
Educación en la Universidad de la Rioja. Actualmente estoy realizando la tesis doctoral con temática relacionada con la educación y las competencias emocionales. Las líneas de investigación desarrolladas son: 1) inteligencia y educación emocional; 2) género y coeducación. Autora de artículos y capítulos de libro relacionados con estas materias. Miembro del G.I de la Universidad de la Rioja: Igualdad y Género. Investigadora del Instituto de Estudios Riojanos. (IER) 